\title{
Research on the Mechanisms of Internal Gear Honing by use of Cone-shape Honing Wheel with Tool Tilt Angle
}

Zheng Guo ( $\sim$ guozheng@xaut.edu.cn )

Xi'an University of Technology

Yan Li

Xi'an University of Technology

Wen-Chao Guo

Xi'an Jiaotong University

Wu-Gang Zhang

Qinchuan Machine Tool \& Tool Corporation, Baoji

\section{Feng Gao}

Xi'an University of Technology

Dong-Ya Zhang

Xi'an University of Technology

\section{Research Article}

Keywords: Honing mechanism, Ring gear , Cone-shape honing wheel , Tool tilt angle

Posted Date: July 19th, 2021

DOl: https://doi.org/10.21203/rs.3.rs-705120/v1

License: (9) (i) This work is licensed under a Creative Commons Attribution 4.0 International License.

Read Full License 


\title{
Research on the mechanisms of internal gear honing by use of cone-shape honing wheel with tool tilt angle
}

\author{
Zheng Guo ${ }^{1}$, Yan Li ${ }^{1}$, Wen-Chao Guo ${ }^{2}$, Wu-Gang Zhang ${ }^{3}$, Feng Gao ${ }^{1}$, Dong-Ya Zhang ${ }^{1}$
}

\begin{abstract}
Ring gear is an important transmission component, but restricted by its ring-shape structure, the tooth surfaces are pretty hard to be finished after heat treatment. Honing is the most commonly used finishing technique for external gear, but for ring gear, it is inconvenient. In this paper, a new type of cone-shape honing wheel is proposed and the honing mechanism is studied for improving the honing performance of ring gear. First of all, the mathematical model of the honing technology by use of cone-shape honing wheel is built, and the merits of the new honing method are discussed. Then, the contact conditions between the honing wheel and the work gear are analyzed, and it is found that the tool tilt angle of the honing wheel has good influences on the honing mark distribution of the work gear. Finally, the honing simulations for ring gear by use of cone-shape honing wheel are carried out, by which the feasibility of the new honing technique are verified.
\end{abstract}

Keywords Honing mechanism • Ring gear • Cone-shape honing wheel $\bullet$ Tool tilt angle

\section{Introduction}

Owing to the merits of higher efficiency, lower cost and better machining quality for hard gears, honing is deemed as an excellent gear finishing technique and is widely used in automotive, aerospace, heavy equipment industries, especially in the areas that require quiet, robust and reliable gearing [1,2]. After years of development, the theoretical and practical application researches of honing technique have achieved great progresses and tend to be perfect, mainly including the topological modification for helical gear, high efficiency honing with high accuracy, electrochemical honing and so on.

Many studies of gear honing are focused on the modification methodology. Tran [3] proposed a new way for longitudinal tooth flank crowning of work gear surfaces in honing process. Han [4] developed a

\footnotetext{
Zheng Guo

guozheng@xaut.edu.cn

Yan $\mathrm{Li}$

glatiagf@163.com 103647

Wen-Chao Guo

wc_guo@163.com

Wu-Gang Zhang

wugang8668@126.com

Feng Gao

gf2713@xaut.edu.cn

Dong-Ya Zhang

dyzhang@xaut.edu.cn

1 School of Mechanical and Precision Instrument Engineering Xi'an University of Technology, Xi'an 710048, China

2 State Key Laboratory for Manufacturing System Engineering, Xi'an Jiaotong University, Xi'an 710049, China

Qinchuan Machine Tool \& Tool Corporation, Baoji 721008, China
}

mathematical model for lead and profile crowning modification of work gear by setting the movement of the honing wheel as two forth-order polynomial functions. Wu [5-8] presented methods for topology modification of helical gears using internal-meshing gear honing. Parallel, many researchers investigate the contact conditions, cutting force vibration, and teeth elastic deformation of honing wheel in the honing process, aiming at improving the honing performance. Klocke [9] carried out the analysis of cutting speed and contact condition of gear honing, forming the basis for optimizing process design. Bagaiskov [10] investigated the sliding parameters and the tooth contact geometry in engagement of gear hone teeth and processed parts with consideration of elastic deformation. Bergs [11] derived a force model of gear honing. Mallipeddi [12] investigated the gear surface characteristics generated by grinding and honing of case hardened steel, figuring out the honed gear has better performance in micro-pitting prevention compared with the ground one. Silva [13] applied particle swarm optimization for achieving the minimum profile error in honing process. In addition, non-conventional honing technique is also the research focus of honing technique. Shaikh [14] investigated the micro-geometry improvement method of straight bevel gears finished by electrochemical honing process and proved that $\mathrm{ECH}$ has great potential to be developed as technologically better, productive, economical alternative to the convention bevel gear finishing processes.

Generally, most of the honing studies are focused on the finish machining of external gear. Due to the ring-shape structure restriction of internal gear, honing technique is merely used in its finish processing. Considering the size of the honing wheel must be 
limited so as to stretch into and hone the whole tooth flank of the work gear, the service life of the honing wheel is discounted compared with the honing wheel for external gear, what's more, in order to avoid the interference between the internal gear and the tool spindle during the honing process, the diameter of the tool spindle should also be limited, so the stiffness of the honing system would be weakened to some extent, which is bad for the finishing quality of the work gear.

Focused on these, an improved honing method for ring gear by use of a new type of cone-shape honing wheel is proposed in this paper. The mathematical model of the new honing technique is built first. Then the contact conditions between the honing wheel and the work gear are analyzed. Finally, the simulations of the honing process for the ring gear are carried out to verifying the honing feasibility and accuracy.
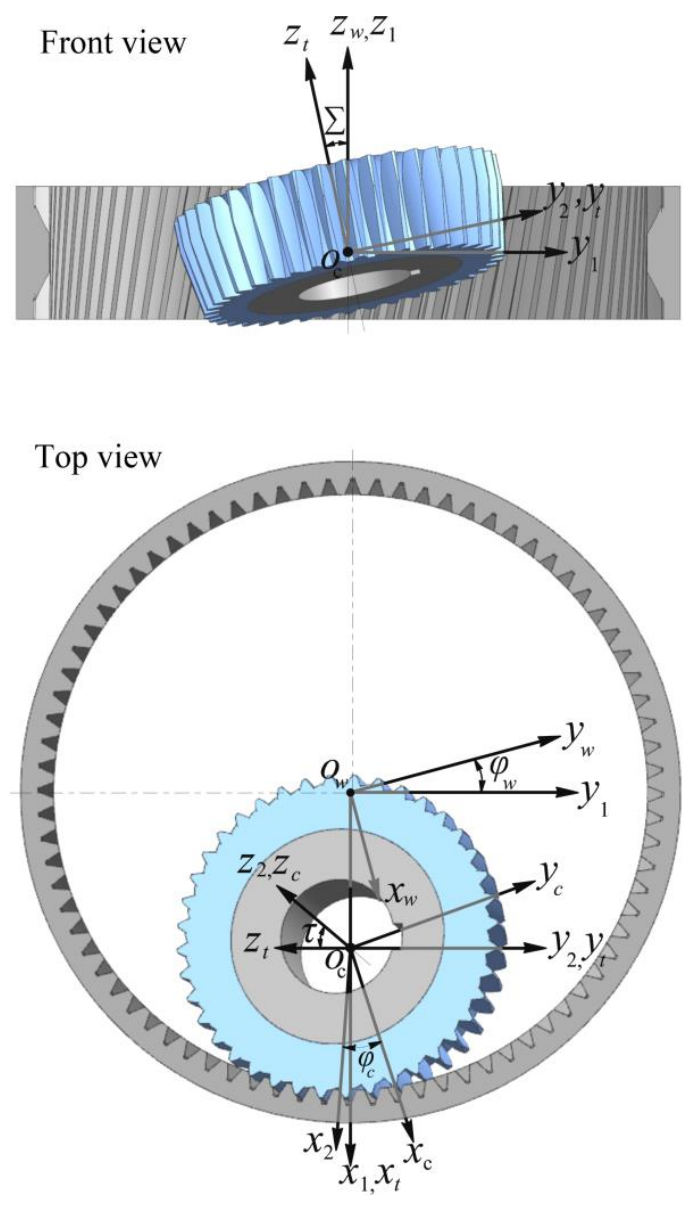

Fig. 1 Machine tool setting of cone-shape honing wheel

\section{Honing principle by use of cone-shape honing wheel}

Several coordinate systems are defined for describing the relative position between the new type cone-shape honing wheel and the work gear as shown in Fig. $1 S_{1}\left(x_{1}, y_{1}, z_{1}\right)$ is the fixed coordinate system of work gear that rotates around and moves along the axis of $z_{1}$, while $S_{w}\left(x_{w}, y_{w}, z_{w}\right)$ is rigidly attached to the work gear. The axial feed velocity of work gear is $\mathbf{F}$, whose direction can be either positive or negative of $z_{1}$. $S_{2}\left(x_{2}, y_{2}, z_{2}\right)$ is the fixed coordinate system of honing wheel that rotates around the axis of $z_{2}$, while $S_{c}\left(x_{c}, y_{c}, z_{c}\right)$ is rigidly attached to the honing wheel. $S_{t}\left(x_{t}, y_{t}, z_{t}\right)$ is the transfer coordinate system. The crossed axes angle and the center distance between the work gear and the honing wheel are $\Sigma$ and a respectively. Besides, there is an additional tool title angle $t$ of the cone-shape honing wheel axis $z_{c}$ relative to the axis $z_{t}$ compared with the original gear honing technology.

The material removal motion is formed by the engagement and the differential movement between the honing wheel and the work gear. The angular speed of the cone-shape honing wheel is defined as $\omega_{c}$ and the axial feed speed of the work gear along $z_{1}$ is $F$, the angular speed of the work gear $\omega_{w}$ can be described as:
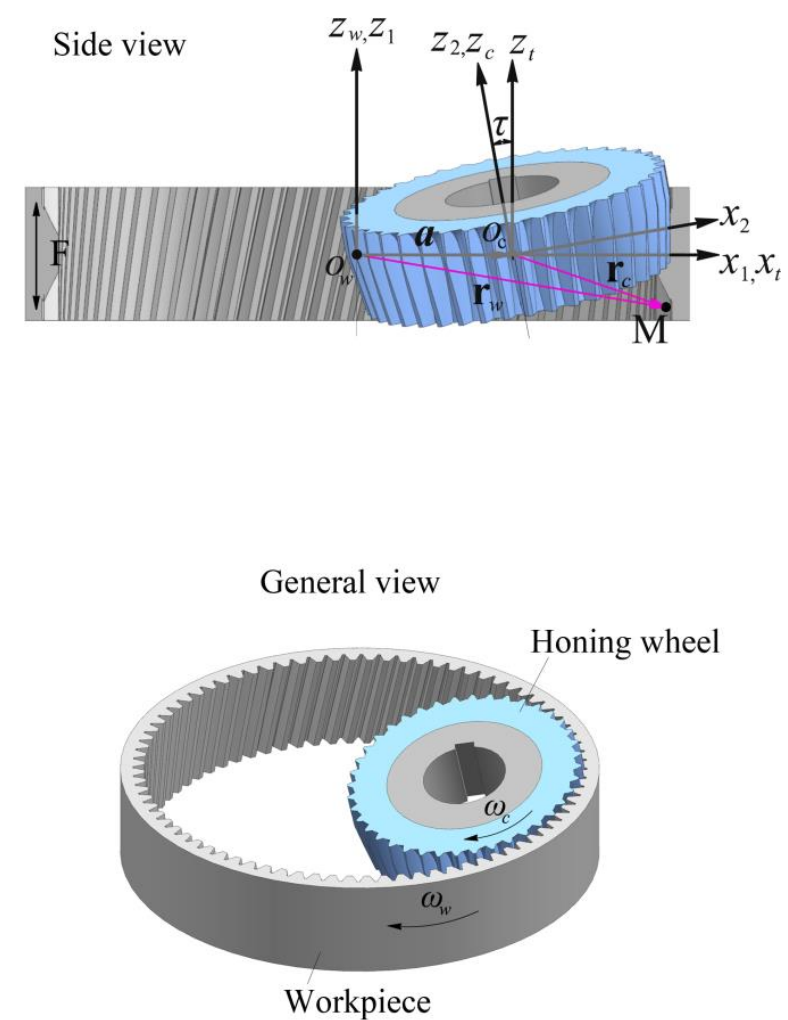

$\omega_{w}=\frac{Z_{c}}{Z_{w}} \omega_{c}+\frac{F}{P}$

Where, $Z_{c}$ and $Z_{w}$ are the numbers of teeth of the honing wheel and the work gear respectively, $P$ is the helix parameter and can be expressed as:

$$
P=\frac{P_{z}}{2 \pi}
$$


Where, $P_{z}$ is the helix lead on the reference cylinder of work gear.

The crossed angle $\Sigma$ between the axes of the honing wheel and the work gear is the sum of the work gear helix angle $\beta_{w}$ and the honing wheel helix angle $\beta_{c}$, and the center distance $\mathrm{a}$ is the difference between the work gear reference radius $r_{w}$ and the honing wheel reference radius $r_{c}$ :

$$
\begin{gathered}
\sum=\beta_{w}+\beta_{c} \\
a=r_{w}-r_{c}=\left(Z_{w}-Z_{c}\right) \cdot m_{t}
\end{gathered}
$$

Where, $m_{t}$ is the transverse module of the work gear.

\section{Mathematical model of cone-shape honing wheel}

The tooth flank of the cone-shape honing wheel can be enveloped by the tooth surface of the work gear. If the target tooth surface of the work gear is involute helicoid, it can be expressed in $S_{w}$ as:

$$
\mathbf{r}_{w}(u, \theta)=\left[\begin{array}{c}
x_{w} \\
y_{w} \\
z_{w} \\
1
\end{array}\right]=\left[\begin{array}{c}
r_{b} \cos (u+\theta)+r_{b} u \sin (u+\theta) \\
r_{b} \sin (u+\theta)-r_{b} u \cos (u+\theta) \\
P \cdot \theta \\
1
\end{array}\right]
$$

Where, $r_{b}$ is the base circle radius of the work gear, $u$ is the involute parameter, $\theta$ is the rotation angle of the transverse tooth profile of the target tooth surface around $z_{w}$.

The normal vector of the involute helicoid can be expressed in $S_{w}$ as:

$$
\mathbf{n}_{w}(u, \theta)=\left[\begin{array}{c}
r_{b} u \sin (u+\theta) \cdot P \\
-r_{b} u \cos (u+\theta) \cdot P \\
r_{b}^{2} u
\end{array}\right]
$$

If the rotation angle of the work gear around axis $z_{1}$ is $\varphi_{w}$, the position coordinates of a point on the target tooth flank can be expressed in $S_{1}$ as:

$$
\mathbf{r}_{w}^{(1)}\left(u, \theta, \varphi_{w}\right)=\mathbf{M}_{1 w} \cdot \mathbf{r}_{w}(u, \theta)
$$

Where, $\mathbf{M}_{1 \mathrm{w}}$ is the transfer-matrix from $S_{w}$ to $S_{1}$, whose upper-left $3 \times 3$ submatrix is $\mathbf{L}_{1 \mathrm{w}}$.

$$
\mathbf{M}_{1 w}\left(\varphi_{w}\right)=\left[\begin{array}{cccc}
\cos \varphi_{w} & -\sin \varphi_{w} & 0 & 0 \\
\sin \varphi_{w} & \cos \varphi_{w} & 0 & 0 \\
0 & 0 & 1 & 0 \\
0 & 0 & 0 & 1
\end{array}\right]
$$

The normal vector can be expressed in $S_{1}$ as:

$$
\begin{aligned}
& \mathbf{n}_{w}^{(1)}\left(u, \theta, \varphi_{w}\right)=\mathbf{L}_{1 w} \cdot \mathbf{n}_{w}(u, \theta)= \\
& {\left[\begin{array}{c}
r_{b} u \sin (u+\theta) \cos \left(\varphi_{w}\right) \cdot P+r_{b} u \sin (u+\theta) \sin \left(\varphi_{w}\right) \cdot P \\
r_{b} u \sin (u+\theta) \sin \left(\varphi_{w}\right) \cdot P-r_{b} u \sin (u+\theta) \cos \left(\varphi_{w}\right) \cdot P \\
u r_{b}^{2}
\end{array}\right]}
\end{aligned}
$$

The angular velocity of the honing wheel $\boldsymbol{\omega}_{\mathrm{c}}$ and angular velocity of the work gear $\boldsymbol{\omega}_{\mathrm{w}}$ can be expressed separately in $S_{1}$ as:

$$
\begin{aligned}
& \boldsymbol{\omega}_{c}^{(1)}=\mathbf{L}_{t 1}^{-1} \cdot \mathbf{L}_{2 t}^{-1} \cdot \boldsymbol{\omega}_{c}= \\
& \omega_{c} \cdot\left[\begin{array}{lll}
-\sin \tau & -\cos \tau \sin \Sigma & \cos \tau \cos \Sigma
\end{array}\right]^{\mathrm{T}} \\
& \boldsymbol{\omega}_{w}^{(1)}=\boldsymbol{\omega}_{w}=i \cdot \omega_{c} \cdot\left[\begin{array}{lll}
0 & 0 & 1
\end{array}\right]^{\mathrm{T}}
\end{aligned}
$$

Where, $i$ is the transmission ratio, $\mathbf{L}_{\mathrm{t} 1}$ is the upper-left $3 \times 3$ submatrix of $\mathbf{M}_{\mathrm{t} 1}$ which is the transfer-matrix from $S_{1}$ to $S_{t}, \mathbf{L}_{2 \mathrm{t}}$ is the upper-left $3 \times 3$ submatrix of $\mathbf{M}_{2 \mathrm{t}}$ which is the transfer-matrix from $S_{t}$ to $S_{2}$ :

$$
i=Z_{c} / Z_{w}
$$

$$
\begin{aligned}
\mathbf{M}_{t 1} & =\left[\begin{array}{cccc}
1 & 0 & 0 & -a \\
0 & \cos \Sigma & \sin \Sigma & 0 \\
0 & -\sin \Sigma & \cos \Sigma & 0 \\
0 & 0 & 0 & 1
\end{array}\right] \\
\mathbf{M}_{2 t} & =\left[\begin{array}{cccc}
\cos \tau & 0 & \sin \tau & 0 \\
0 & 1 & 0 & 0 \\
-\sin \tau & 0 & \cos \tau & 0 \\
0 & 0 & 0 & 1
\end{array}\right]
\end{aligned}
$$

The relative velocity of the point on the target tooth flank around $z_{1}$ relative to the honing wheel around $z_{2}$ can be expressed in $S_{1}$ as:

$$
\begin{aligned}
& \mathbf{v}_{c w}^{(1)}=\mathbf{v}_{c}^{(1)}-\mathbf{v}_{w}^{(1)}=\boldsymbol{\omega}_{c}^{(1)} \times\left(\mathbf{r}_{w}^{(1)}-\mathbf{a}\right)-\boldsymbol{\omega}_{w}^{(1)} \times \mathbf{r}_{w}^{(1)}= \\
& \omega_{c} \cdot\left[\begin{array}{c}
x_{w} P_{1} \sin \varphi_{w}+y_{w} P_{1} \cos \varphi_{w}+z_{w} \sin \Sigma \cos \tau \\
-x_{w} P_{1} \cos \varphi_{w}+y_{w} P_{1} \sin \varphi_{w}-z_{w} \sin \tau+a \cos \Sigma \cos \tau \\
x_{w} P_{2}+y_{w} P_{3}+a \sin \Sigma \cos \tau
\end{array}\right]
\end{aligned}
$$

Where, $r_{w}$ turn into upper $3 \times 1$ submatrix of itself, and $a$ is the position vector of the honing wheel:

$$
\begin{gathered}
\mathbf{a}=a \cdot\left[\begin{array}{lll}
0 & 0 & 1
\end{array}\right]^{\mathrm{T}} \\
P_{1}=\cos \Sigma \cos \tau-i \\
P_{2}=\sin \varphi_{w} \sin \tau-\cos \varphi_{w} \sin \Sigma \cos \tau \\
P_{3}=\cos \varphi_{w} \sin \tau+\sin \varphi_{w} \sin \Sigma \cos \tau
\end{gathered}
$$

The constraint of the point on the target tooth flank being a meshing point in $S_{1}$ is that the normal vector $\mathbf{n}_{\mathrm{w}}^{(1)}$ is vertical with the relative velocity $\mathbf{v}_{\mathrm{cw}}^{(1)}$ :

$$
\mathbf{n}_{w}^{(1)}\left(u, \theta, \varphi_{w}\right) \cdot \mathbf{v}_{c w}^{(1)}\left(u, \theta, \varphi_{w}\right)=0
$$

The meshing point $\mathbf{r}_{\mathrm{M}}^{(1)}(\mathrm{u}, \theta)$ in $S_{1}$ can be obtained by solving the Eqs. (7) and (17) simultaneously, so the position vector of the point on the tooth flank of the honing wheel can be expressed in $S_{c}$ as:

$$
\mathbf{r}_{c}\left(u, \theta, \varphi_{c}\right)=\left[\begin{array}{llll}
x_{c} & y_{c} & z_{c} & 1
\end{array}\right]^{\mathrm{T}}=\mathbf{M}_{c 2}\left(\varphi_{c}\right) \cdot \mathbf{M}_{2 t} \cdot \mathbf{M}_{t 1} \cdot \mathbf{r}_{M}^{(1)}
$$

Where, $\mathbf{M}_{\mathrm{c} 2}$ is the transfer-matrix from $S_{2}$ to $S_{c}$ : 


$$
\mathbf{M}_{c 2}\left(\varphi_{c}\right)=\left[\begin{array}{cccc}
\cos \varphi_{c} & \sin \varphi_{c} & 0 & 0 \\
-\sin \varphi_{c} & \cos \varphi_{c} & 0 & 0 \\
0 & 0 & 1 & 0 \\
0 & 0 & 0 & 1
\end{array}\right]
$$

Based on the parameters of the tool and the work gear shown in Table 1, the geometric model of the cone-shape honing wheel is built. Due to the tool title angle $t$ attached to the honing wheel, the shape of the honing wheel is changed into cone-shape, which is composed of a conical segment $b_{1}$ and a cylindrical segment $b_{2}$, as shown in Fig 2(a). The teeth of the conical segment of the honing wheel are meshing with the teeth of the work gear during the honing process, while the teeth of the cylindrical segment are not, which acts as the spare dressing segment of the honing wheel. By use of the new type cone-shape honing wheel, the honing performance of ring gear can be improved to some extent.

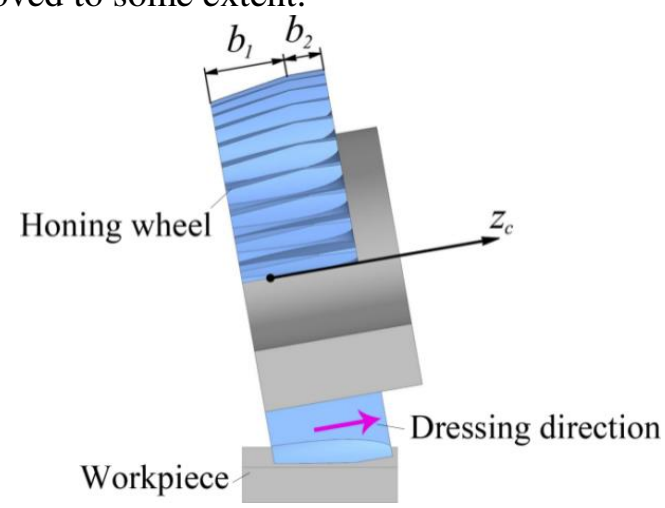

(a) Cone-shape honing wheel model

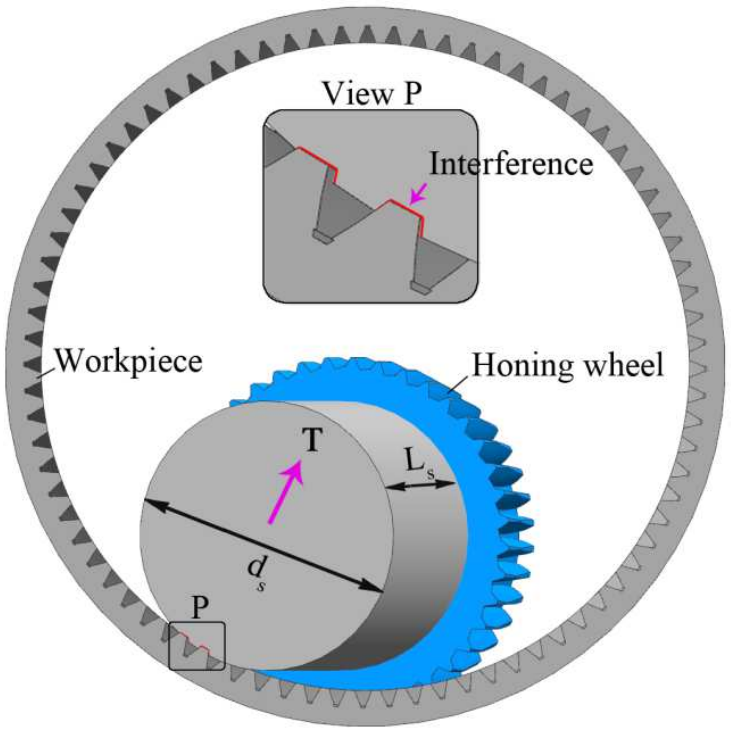

(b) Honing interference

Fig. 2 Geometrical model of cone-shape honing wheel

Firstly, different from the conventional radial direction dressing method that results in the tooth shape change of the honing wheel just as the displacement effect on gears, the dressing direction of the cone-shape honing wheel is axial, which will not result in the teeth shape change of the honing wheel for each dressing procedure, so it is good for the consistence of the honing accuracy of the work gears. What's more, the total available dressing times of the cone-shape honing wheel is mainly decided by the width of the cylindrical part, so the total available dressing times can be increased according to the actual demand by widening the cylindrical segment $b_{2}$. For example, if the width of the cylindrical segment $b_{2}$ is increased form $10 \mathrm{~mm}$ to $30 \mathrm{~mm}$, and the dressing amount for each time is $1 \mathrm{~mm}$, the total available dressing times can be increased from 10 to 30 .

Secondly, owing to the tool tilt angle of the honing wheel, the interference between the tool spindle of honing wheel and the work gear, as shown in Fig. 2(b) view $\mathrm{P}$, can be avoided more easily. For example, in order to avoiding the interaction, the tool spindle diameter $d_{s}$ should be less than $41 \mathrm{~mm}$, while if the tool tilt angle, lifted up in the T direction, is increased to 10 degrees, the maximum tool spindle diameter $d_{s}$ can be increased to $50 \mathrm{~mm}$, so larger diameter tool spindle can be used, which is good for the stiffness of the honing system.

Thirdly, the honing velocity distribution can be affected by the tool tilt angle of the honing wheel, which could have a good effect on the honing performance for ring gear. The detailed analysis is carried out in section 4 .

\section{Contact analysis of honing process}

The contact analysis is carried out to investigate the honing velocity distribution and the contact condition between the cone-shape honing wheel and the work gear. The basic parameters of the tool and the internal gear are shown in Table 1. For each rotation angle $\varphi_{w}$ of work gear, there will be a contact line between the work gear tooth flank and the honing wheel tooth flank, which can be expressed by the Eq. (20) in $S_{w}$. The sliding velocity of the meshing point on the work gear tooth flank relative to the honing wheel can be expressed by the Eq. (21) in $S_{w}$ [15]:

$$
\begin{aligned}
& \mathbf{r}_{M}^{(w)}\left(u, \theta, \varphi_{w}\right)=\mathbf{M}_{w 1}\left(\varphi_{w}\right) \cdot \mathbf{r}_{M}^{(1)} \\
& \mathbf{v}_{c w}^{(w)}\left(u, \theta, \varphi_{w}\right)=\mathbf{L}_{w 1}\left(\varphi_{w}\right) \cdot \mathbf{v}_{c w}^{(1)}
\end{aligned}
$$

Table 1 Parameters of the gear and the honing wheel

\begin{tabular}{lcc}
\hline Parameters of internal gear & & \\
Number of teeth & $Z_{w}$ & 80 \\
Normal module & $m$ & 2.25 \\
Pressure angle & $\alpha$ & $22.5^{\circ}$ \\
Helix angle & $\beta_{w}$ & $12^{\circ}$ \\
Revolution speed & $\omega_{w}$ & $1200 \mathrm{r} / \mathrm{min}$ \\
Parameters of honing wheel & & \\
Number of teeth & $Z_{c}$ & 40 \\
Helix angle & $\beta_{c}$ & $0^{\circ}$ \\
Tool tilt angle & $\tau$ & $10^{\circ}$ \\
\hline & &
\end{tabular}


The contact lines between the work gear and the honing wheel at different meshing positions are shown in Fig.3. As shown in the figures, groups of contact lines with different length and unanimous directions can be joined up to the meshing zones on the tooth flanks of work gear and honing wheel. With the differential motion of the work gear, the whole flanks of the work gear can be enveloped. In order to distinguish the two sides of the teeth of the work gear, the front flank of the tooth space, referring to the rotation direction, is defined as the approach flank " $\mathrm{A}$ ", while another flank of the tooth space is defined as the recess flank "R".

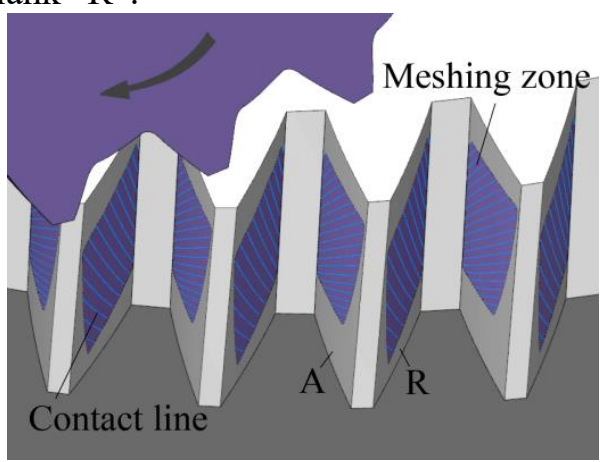

(a) Work gear

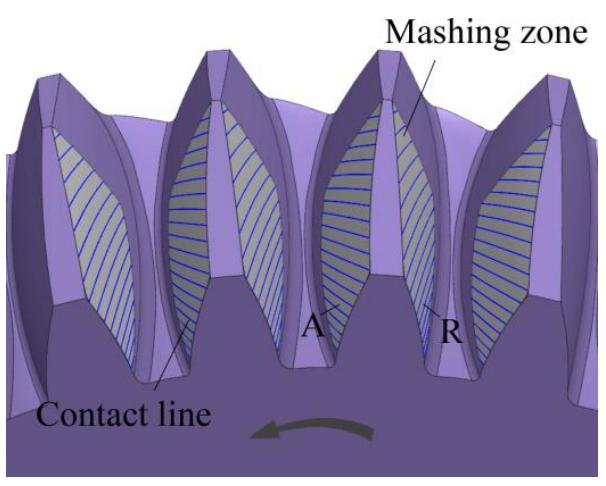

(b) Honing wheel

Fig. 3 Contact lines and meshing zones

The honing velocities of the meshing points on the contact lines of the work gear can be calculated by using the Eq. (21). The theoretical calculation results of the contact patterns and honing velocity distributions with different tool tilt angle on the tooth flanks of the work gear are shown in Fig. 4. As shown in figure, when the tool tilt angle is set to be zero, the honing velocities are regularly distributed on the tooth flanks, which are identical with the original honing method. The directions of the honing velocities on the addendum are obviously different from the dedendum, which can be separated by the reference lines RL near the pitch circle. So the tiny honing marks left on the tooth flanks will be unevenly distributed: the directions of the honing marks on the addendum and dedendum are greatly different from the directions near the pitch circle, which is not good at the tooth contact fatigue life of the work gear [12].

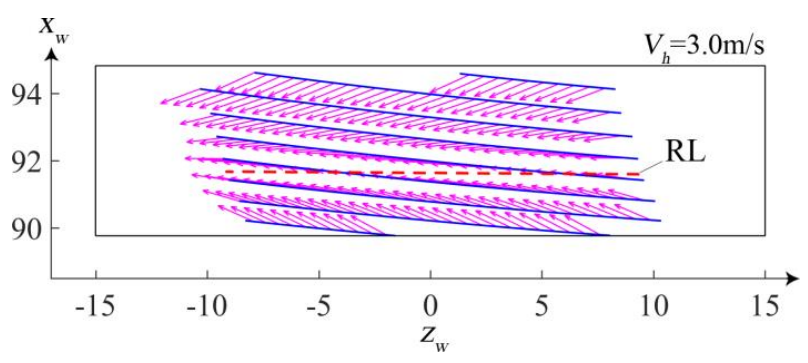

(a) Approach flank velocity distributions when $\tau=0^{\circ}$

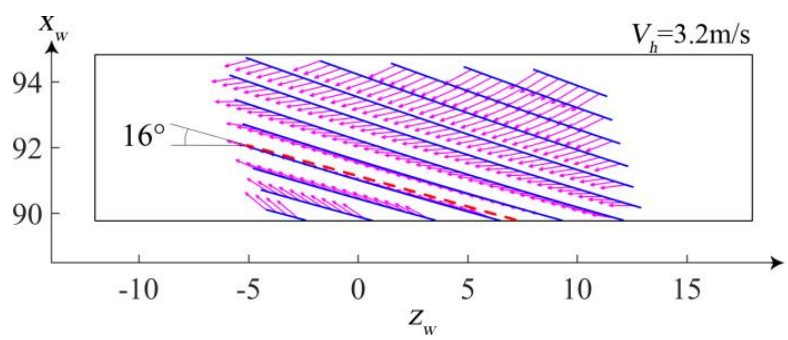

(b) Approach flank velocity distributions when $\tau=5^{\circ}$

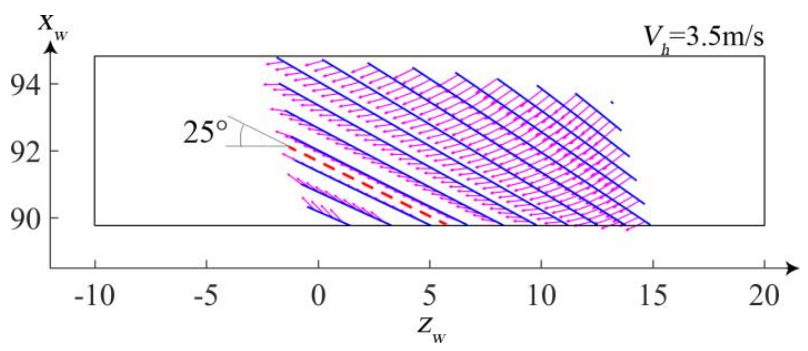

(c) Approach flank velocity distributions when $\tau=10^{\circ}$

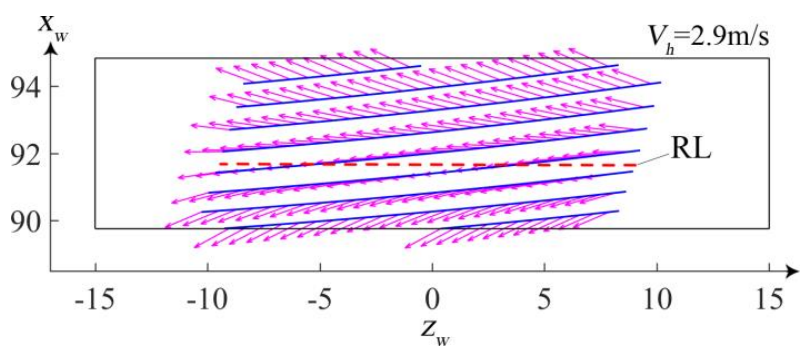

(d) Recess flank velocity distributions when $\tau=0^{\circ}$

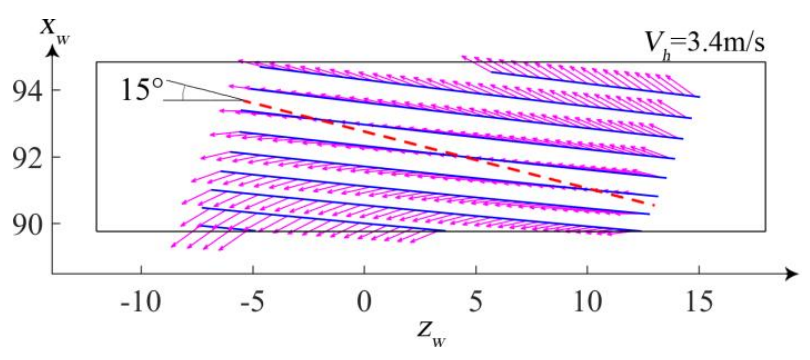

(e) Recess flank velocity distributions when $\tau=5^{\circ}$

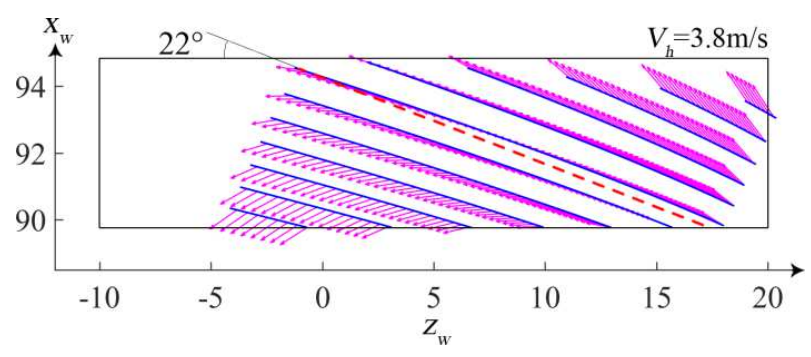

(f) Recess flank velocity distributions when $\tau=10^{\circ}$

Fig. 4 Honing velocity distributions with different tool tilt angle $\boldsymbol{\tau}$ 
When the tool tilt angle is increased to 5 degrees, the honing velocity distribution on the tooth flanks have changed somewhat compared with the zero degree condition. While when the tool tilt angle is continuously increased to 10 degrees, the distribution of honing velocity is changed evidently: the honing velocities of the right side of the approach flank and the left side of the recess flank mostly incline to the dedendum, while the opposite sides are mainly toward to the dedendum, and the directions of the reference lines "RL" and the average honing speeds $V_{h}$ of the points on the meshing zone are also changed to some extent. As shown in Fig. 4, when the tool tilt angle is increased from 0 to 10 degrees, the inclination angle of the reference line of the approach flank increases from 0 to 25 degrees and the average honing speed rises from 3.0 to $3.5 \mathrm{~m} / \mathrm{s}$. Thus, it can be considered that the tool tilt angle of honing wheel has evident influence on the honing velocity distribution compared with the original honing method, which would have the effect of homogenizing the honing marks left on the teeth flanks of the work gear with axial differential motion in the honing process. So it can be concluded that the tool tilt angle of the honing wheel has good influences on the honing mark distribution, which is good for the honing performance and the service life of the work gear.

\section{Honing simulation and verification}

In actual, the machining accuracy of the work gear is inevitably affected by the cutting tool accuracy, machine tool accuracy, thermal deformation of machine tool and so on. In order to eliminate the influences of all these factors on the honing precision verification, machining simulation is carried out so as to verifying the feasibility of ring gear honing by use of the cone-shape honing wheel. The five axis honing machine tool model is built as shown in the Fig. 5. The $\mathrm{X}, \mathrm{Y}$ and $\mathrm{Z}$ axes are the three orthogonal moving axes whose positive directions defined as the Cartesian coordinate system. The A axis is the swing axis that rotates around $\mathrm{X}$ axis. The $\mathrm{C}$ and the $\mathrm{C}_{1}$ axes are the work gear rotation axis and the honing wheel rotation axis respectively, which are coupled together by electronic gearbox.

The machining allowance of the work gear is $d$ as shown in Fig. 5(b). The relative position and orientation between the honing wheel and the work gear are set based on the parameters shown in Table 1 . The cone-shape honing wheel and the work gear rotate around their own axis according to the transmission ratio $i$. With the axial differential motion of the work gear, the whole tooth flanks of the work gear can be honed, as shown in Fig. 5(c). The comparison between the honed tooth flanks and the designed tooth flanks are shown in Fig. 5(d), the result shows that the honing allowance is removed completely and the honed tooth flanks are smooth enough which coincide with the designed tooth flanks very well.
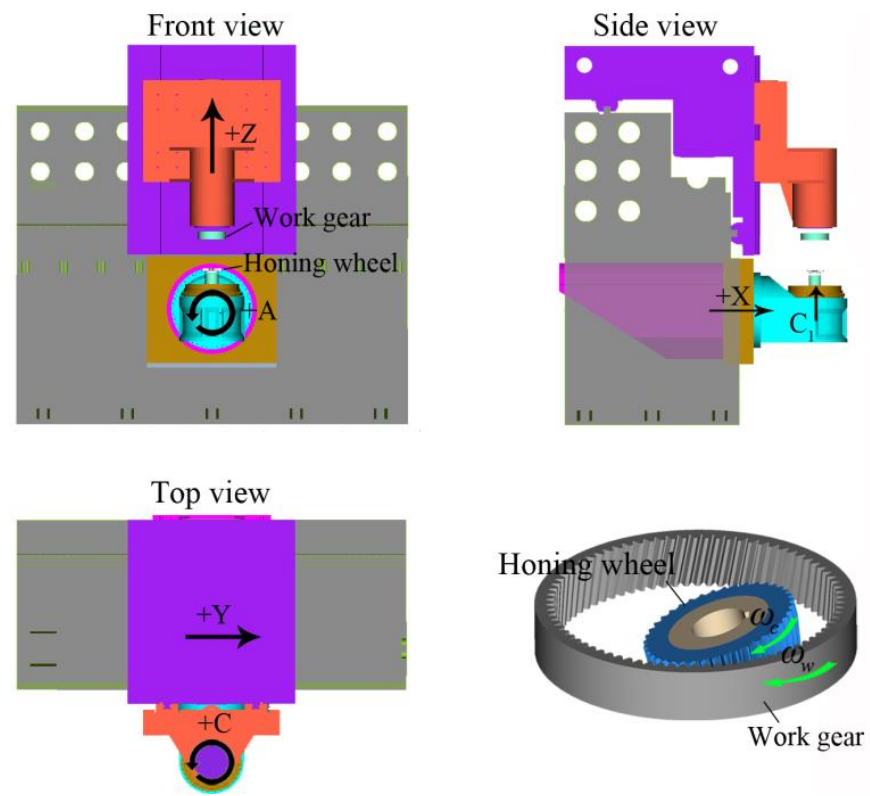

(a) Three views of honing machine tool model

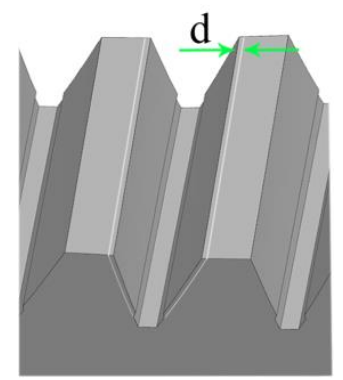

(b) Machining allowance (c) Finished tooth flanks

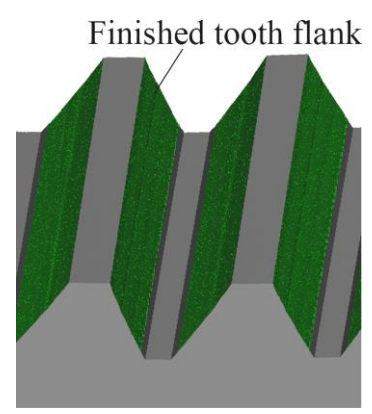

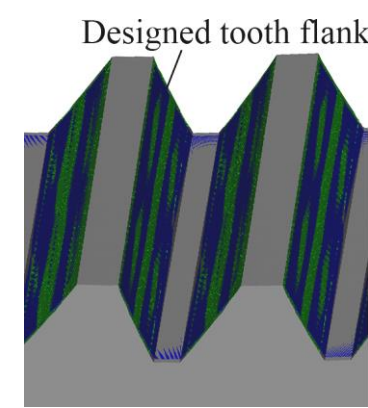

(d) Flanks comparison
Fig. 5 Honing movement simulation

Aiming at testing the honing accuracy, further simulation analysis is carried out. The model of the cone-shape honing wheel, as shown in Fig. 6(a), are built by applying the point set of the tooth flank that can be obtained by solving the above-mentioned tooth flank equations. The machining allowance of the work gear is $d$. The relative position and orientation between the honing wheel and the work gear are set based on the parameters shown in Table 1. In the process of honing simulation, the tooth flanks of the work gear are generated by Boolean calculation between the blank workpiece and the cone-shape honing wheel at each meshing position, so the tooth flanks of the work gear 
are composed of a series of striped marks left by the teeth of the honing wheel, as shown in Fig. 6(b). If the theoretical calculation is correct, the marks should be tangent to the theoretical tooth flanks of the work gear and the tangent lines coincide with the theoretical contact lines. In order to verify this, the tooth profile deviation of the work gear should be obtained, so the coordinates of several points on the transverse tooth profiles of the work gear are measured as shown in Fig. 6(c), and then the comparison between the tooth profiles and the standard involutes are conducted. Reference [16,17] shows the algorithms of profile deviation calculation.

The calculation results of tooth profile deviation are shown in Fig. 6(d,e). The measurement results show that the transverse tooth profiles of the approach flank and the recess flank of the work gear are composed of a series of small arcs which correspond with the marks on the tooth flanks of the work gear generated by the teeth of the honing wheel in the honing simulation. The current tooth profile deviation mostly reflect the height of the marks, which related with and can be decreased by minimizing the simulation steps, so the fluctuation of the lowest points of the marks, approximately one micron, represent the tooth profile deviation best. Considering the modeling accuracy of UG software is about one micron as well, the tooth profile of the honed work gear can be considered as accurate.

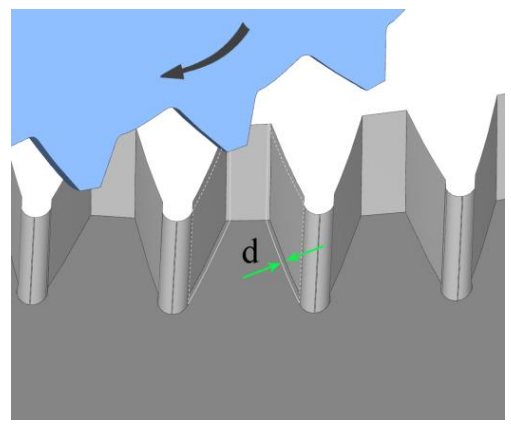

(a) Honing wheel and work gear

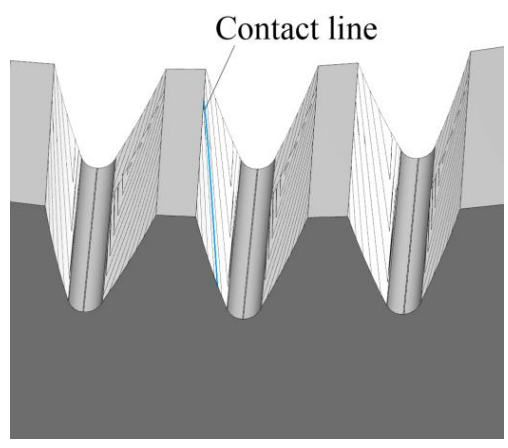

(b) Finished tooth flanks

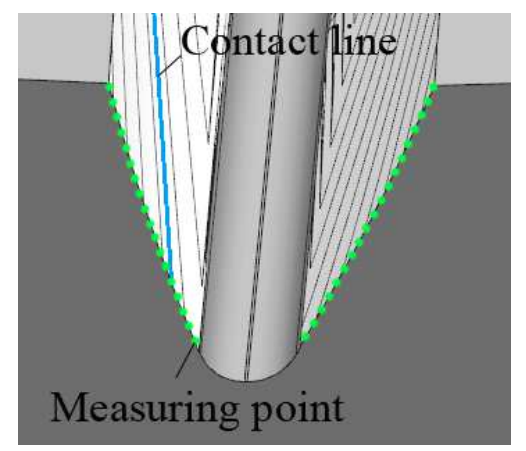

(c) Transverse tooth profiles

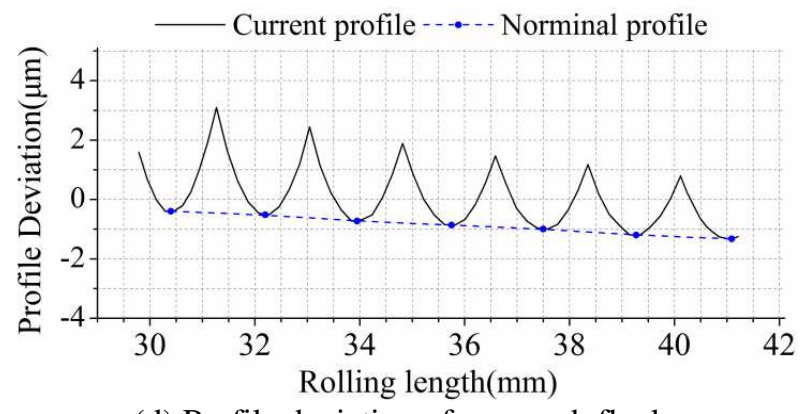

(d) Profile deviation of approach flank

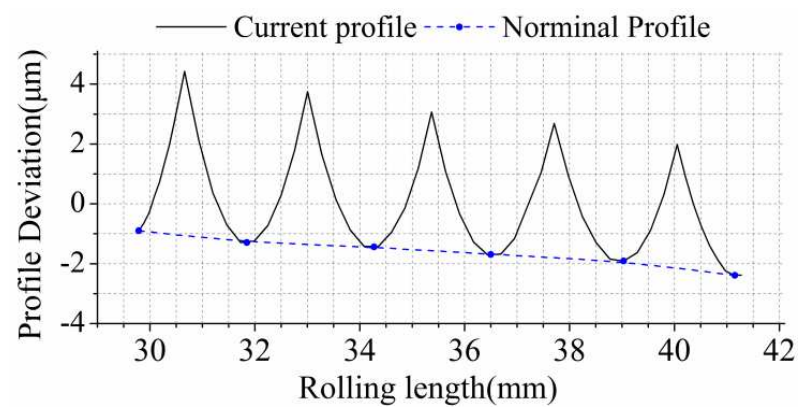

(e) Profile deviation of recess flank

Fig. 6 Honing accuracy simulation

\section{Conclusion}

An improved honing method by use of new type cone-shape honing wheel with tool tilt angle is proposed, by which the total available dressing times of the honing wheel for ring gear, with stable honing accuracy, can be increased, and the interference between the tool axis and the work gear can be avoided more easily.

The mathematical model of the new honing technology by use of cone-shape honing wheel is built, and the contact analysis between the honing wheel and the ring gear is carried out. The results show that the honing velocity distribution can be affected by the tool tilt angle of the honing wheel, which could have a good effect on the honing performance for ring gear.

The simulation and verification for the new type honing method for ring gear are carried out, by which the feasibility of the honing movement are verified, and the tooth profile of the honed work gear is proved to be accurate. 


\section{Declarations}

\section{Fundings}

The authors gratefully acknowledge this project funded by China Postdoctoral Science Foundation (CPSF No.2020M673602XB), National Natural Science Foundation of China (NSFC No. 51775432) and Shaanxi Province Major Science and Technology Project (SPMST No. 2018ZDZX01-02-01).

\section{Conflicts of interest}

The authors declare no conflicts of interest.

\section{Availability of data and material}

The datasets used or analysed during the current study are available from the corresponding author on reasonable request.

\section{Code availability}

Not applicable.

Ethical approval

Not applicable.

\section{Consent to participate}

Not applicable.

\section{Consent to publish}

Not applicable.

\section{Author contribution}

Zheng Guo: General concept and original draft preparation; writing; and editing. Yan Li: Introduction; review; and editing. Wen-Chao Guo: Honing simulation by use of the cone-shape honing wheel. Wu-Gang Zhang: Further simulation of honing and measuring. Feng Gao: Calculation of tooth profile deviation. Dong-Ya Zhang: Preparation of conclusions and editing.

\section{References}

[1] Garshin A, Fedotova S (2008) Abrasive materials and tools, manufacturing procedure: instructional aid. Polytechnic University Press, New York, US.

[2] Dos Santos SP, Brandão LC, Gallicchio LH, Silveira ZC (2012) Finishing process analysis between honing and hard hobbing in pinion gears applied to a steering system. Energy Procedia 14: 2-8.

[3] Tran VT, Hsu RH, Tsay CB (2015) A methodology for longitudinal tooth flank crowning of the helical gear on a CNC honing machine. Advanced Materials Research 1091: 53-62.

[4] Han J, Zhu Y, Xia L, Tian X (2018) A novel gear flank modification methodology on internal gearing power honing gear machine. Mechanism and Machine Theory 121: 669-682.

[5] Tran VQ, Wu YR (2020) A novel method for closed-loop topology modification of helical gears using internal-meshing gear honing. Mechanism and Machine Theory 145: 1-15.

[6] Tran VQ, Wu YR (2018) Dual lead-crowning for helical gears with long face width on a CNC internal gear honing machine. Mechanism and Machine Theory 130: 170-183.
[7] Wu YR, Tran VT (2016) Transmission and load analysis for a crowned helical gear pair with twist-free tooth flanks generated by an external gear honing machine. Mechanism and Machine Theory 98: 36-47.

[8] Wu YR, Tran VT (2015) Lead crowning and anti-twist for tooth flank of a heat treated helical gear on internal CNC honing machine. Applied Mechanics and Materials 799-800: 554-559.

[9] Klocke F, Gorgels C, Vasiliou V (2009) Analysis of the influence of gear dimensions on cutting speed and contact conditions during the gear honing process. Production Process 3(3): 255-259.

[10] Bagaiskov Y (2019) Study of sliding parameters in engagement of gear hone teeth and processed parts with consideration of elastic deformation. Materials Today: proceedings 19: 2262-2265.

[11] Bergs T (2018) Cutting force model for gear honing. CIRP Annals-Manufacturing Technology 67: 53-56.

[12] Mallipeddi D, Norell M, Sosa M, Nyborg L (2019) The effect of manufacturing method and running-in load on the surface integrity of efficiency tested ground, honed and superfinished gears. Tribology International 131: 277-287.

[13] Da Silva S.P, Filho S.L.M.R, Brandão L.C (2014) Particle swarm optimization for achieving the minimum profile error in honing process. Precision Engineering 38 (4): 759-768.

[14] Shaikh J.H, Jain N.K (2016) Investigations on micro-geometry improvement of straight bevel gears finished by electrochemical honing process. The International Journal of Advanced Manufacturing Technology 85(9-12): 2223-2234.

[15] Litvin F. L, Gonzalez-Perez I, Fuentes A, Vecchiato D, Sep T.M (2005) Generalized concept of meshing contact of involute crossed helical gears and its application. Computer Methods in Applied Mechanics and Engineering 194 (34-35): 3710-3745.

[16] Zheng FY, Zhang MD, Zhang WQ, Guo XD (2018) Research on the tooth modification in gear skiving. journal of mechanical design 140(8): 084502-1-9.

[17] Guo Z, Mao SM, Du XF, Ren ZY (2017) Influences of tool setting errors on gear skiving accuracy. The International Journal of Advanced Manufacturing Technology 91(9-12): 1-9. 